\title{
Assessment of Weight Loss in the Management of Patients with Type 2 Diabetes Mellitus in Primary Care in Trinidad
}

Kameel Mungrue*, Lesley-Ann Roper and Tamika Chung

Public Health \& Primary Care, Faculty of Medical Sciences, University of the West Indies, EWMSC, Mt Hope, Trinidad

\begin{abstract}
Objectives: To determine the proportion of patients with Type 2 diabetes mellitus (T2DM) who lost 5-10\% of their body weight as part of their initial management. To determine associations between metformin use, advice on dietary and physical modification on weight loss.

Design and method: A retrospective observational study was performed with a total of 647 adults age range 71years (92-21) with T2DM, identified from the diabetic registry at El Socorro health center. Adults with BMI>25, demographics, initial weight on joining clinic and weight 12-18 months after attending clinic, lifestyle changes and metformin use were recorded. Data was analyzed using SPSS version 15. Multiple linear regression was used to determine predictors of weight loss. Additionally, any relationships between variables and weight loss were highlighted
\end{abstract} using the Pearson's correlation.

Results: $410(63.4 \%$ ) of the 647 participants met the criteria for entry into the study which meant that at entry $63.4 \%$ of patients with T2DM were overweight or obese. Further 206(50.2\%) participants gained weight, 196(47.8\%) lost weight and $8(2 \%)$ had no change. The 196 participants who lost weight had a mean weight loss of $3.9 \mathrm{kgs}$ of which only $62(15 \%)$ achieved at least $5 \%$ weight loss. The use of both lifestyles interventions and metformin produced the largest weight reduction $(11.8 \mathrm{Kgm})$, which supports the ADA recommendation as the current method of initiating treatment in the patient with T2DM.

Conclusion: Only $15 \%$ of adults lost at least $5 \%$ of their initial weight according to ADA recommendations. With $51 \%$ of adults gaining weight it is recommended that more emphasis should be placed on weight loss as part of initial management of diabetes in public health settings.

\section{Introduction}

Type 2 diabetes mellitus (T2DM) is a chronic disease characterized by $\beta$ cell dysfunction with relative rather than absolute insulin deficiency and insulin resistance. [1] The World Health Organization (WHO) estimates that more than 180 million people worldwide have diabetes. [2] T2DM is expected to reach pandemic levels, rising from 171 million in 2000 to 366 million in 2030. In the developing world the numbers are expected to rise from 84 million to 228 million during the same time period. [3]

$\mathrm{T} 2 \mathrm{DM}$ is the most common presentation of the disease accounting for almost $90 \%$ of all diabetes cases worldwide [2]. In 2005, an estimated 1.1 million people died from diabetes worldwide [2]. In Trinidad, the prevalence of type 2 diabetes was estimated at 47 per 1000 in males and 58 per 1000 in females. [4] The death rate per 100000 population for diabetes mellitus in Caribbean increased from 29.3 in 1995 to 54.9 in 2000 as reported by the Caribbean Epidemiological Centre (CAREC) a regional surveillance unit. [5] In addition Alberto et al estimated that the total annual cost associated with diabetes and its complications was US\$65.2 billion for Caribbean and Latin American countries in 2000. [6] The impact on health services is overwhelming in spite of evidence provided by Wagner et al in 2001 that a reduction in utilization of health services occured when hemoglobin $A_{1 C}$ was reduced by $1 \%$ in patients in the United States. [7]

In 2002 Gulliford and Mahabir reported that $49 \%$ of patients with diabetes in Trinidad have peripheral neuropathy, $12 \%$ have a foot ulcer and 4\% have had an amputation. [8] The Diabetes Control and Complications Trial (DCCT) and the UKPDS showed that near normal glycaemia reduces the development and progression of microvascular and neuropathic complications by approximately 50\% in T2DM $[9,10]$
Thus alleviating the economic and social burdens associated with diabetic complications. Therefore a concerted effort by all stakeholders especially primary care physicians is necessary to ensure the delivery and sustainability of quality health services towards the attainment of glycaemic control.

The American Diabetic Association (ADA) and the European Association for the study of Diabetes (EASD) published an expert consensus statement on the approach to management of hyperglycemia in individuals with type 2 diabetes. [11] These guidelines recommend intervention at the time of diagnosis with metformin in combination with lifestyle changes (MNT-medical nutriton therapy and exercise). In the Caribbean "The Manual for Managing Diabetes in Primary Care" also recommends an initial non-pharmacological management of hyperglycemia by diet and physical exercise for overweight and obese patients. This requires initial assessment of nutritional status using either BMI or waist circumference, followed by dietary advice and referral to a nutritionist / dietitian, and emphasis on regular physical activity. If diet and exercise do not achieve glycaemic control (HbAlc

${ }^{*}$ Corresponding author: Kameel Mungrue, Public Health \& Primary Care, Faculty of Medical Sciences, University of the West Indies, EWMSC, Mt Hope, Trinidad, Tel: 868645 6741; E-mail: kmungrue@fms.uwi.tt

Received December 13, 2010; Accepted March 04, 2011; Published March 06 , 2011

Citation: Mungrue K, Roper LA, Chung T (2011) Assessment of Weight Loss in the Management of Patients with Type 2 Diabetes Mellitus in Primary Care in Trinidad. J Diabetes Metab 2:120 doi:10.4172/2155-6156.1000120

Copyright: (c) 2011 Mungrue K, et al. This is an open-access article distributed under the terms of the Creative Commons Attribution License, which permits unrestricted use, distribution, and reproduction in any medium, provided the original author and source are credited. 
$\leq 7 \%)$, metformin is indicated as an adjunct to therapy. [12] Lee et al. [13] showed that metformin decreases calorie intake and leads to a reduction in body weight. [13] However, Avenel et al [14] showed that metformin did not affect body weight or body fat mass although leptin concentrations were significantly reduced. [14] The United Kingdom Prospective Diabetic Study (UKPDS, 1998) showed that relative to conventional treatment with diet, treatment with metformin resulted in significant reductions in the risk of any diabetes complication, diabetesrelated death, all-cause mortality, and myocardial infarction. [10]

One of the most important modifiable risk factors in T2DM is body weight. [15] The World Health Organization defines overweight as a body mass index of 25.029.9 and obesity as a body mass index $>30$. [16] Obesity complicates the management of type 2 diabetes by increasing insulin resistance and thereby increasing blood glucose concentrations. It is also an independent risk factor for dyslipidaemia, hypertension and cardiovascular disease and increases the risk of cardiovascular complications and mortality in patients with type 2 diabetes. [17] It has been shown that prevention and treatment of obesity will help in the management and treatment of diabetes and is especially recommended for all overweight or obese adults who have T2DM. [18]

Hussain et al. [15] showed that almost $90 \%$ of T2DM is attributable to excess weight. [15] Aucott et al. [19] found that relative risk for morbidity and mortality in patients with a body mass index (BMI) of $\geq 28$ was reduced by as much as $25 \%$ if there was intentional weight loss. [19] In fact losing between 9 to 13 kilograms was the most protective, but even modest weight loss improved the metabolic control of glucose. [19] Notwithstanding the aim should be for a gradual and modest weight loss of $510 \%$ of initial body weight by caloric restriction, increased physical activity, and behavior therapy. [20] Short term studies have shown that even modest weight lost (5-10\% of initial body weight) in T2DM improves blood pressure, lipid concentrations, insulin sensitivity and glycaemic control and can even reduce the need for diabetic medications. [20] By reducing these risk factors, weight loss may reduce the high risk of vascular complications and death among patients with diabetes [18].

The aim of this study is to determine the proportion of patients with T2DM who lost 5-10\% of their body weight as part of their initial management. The study focuses on patients in the public health setting. Additionally, any associations between advice on dietary and physical modification from the health practitioner and metformin use were investigated.

\section{Methods}

A cross-sectional observational study design was used in the assessment of weight loss as part of the initial management of T2DM. We first developed a diabetic registry from clients in a primary care setting. All clients who meet the WHO criteria for T2DM were eligible for entry into the database. All records were subsequently reviewed and patients who met the following inclusion criteria were selected for the study: T2DM, Age $\geq 20$ years, attendance at a primary care facility for $\geq$ 1year, overweight or obese (WHO criteria) [16].

A data collection instrument was designed and validated to collect data which were stored, retrieved and analysed using SPSS version 15.0 The data collected included demographics (age, gender), date, height and weight at initiation of management, weight after at least 1 year of management at the facility, use of metformin and documented lifestyle advice on diet/exercise which included; (1) reduction in total fat and saturated fat intake and avoid trans fat, (2) taught healthy eating, carbohydrate awareness or carbohydrate counting to match medication and level of detail the patient can handle, (3) individualize meal plans to allow for social and cultural influences, eating habits and readiness to change and (4) promote regular physical activity. The main outcome variable measured was percentage change in weight over a 12-18 month time period. [21]

Statistical analysis included multiple linear regression to determine if age, initial weight, use of Metformin or advice on diet and exercise was a predictor of weight loss. In addition the difference in mean weight loss in patients with no intervention, diet and exercise only, metformin use only and diet and exercise and metformin use were tested using oneway analysis of variance. Multiple comparisons were performed using Scheffess test. A Pearson's correlation was also performed to identify an association between age and weight loss. Results were presented as means, unless otherwise stated. A $P$ values $\leq 0.05$ was considered significant. This study protocol was approved by Ethics committee of the Faculty of Medical Sciences, of the University of the West Indies.

\section{Results}

The number of patients entered into the diabetic registry was 647 of which 410 patients met all inclusion criteria. In other words $410(63.4 \%)$ participants were either overweight or obese on entry into the study and $247(36.6 \%)$ were not. There were twice as many females $(274$ $66.8 \%)$ than males $(136,33.2 \%)$ reflecting the typical pattern of gender disparity in the occurrence of T2DM in Trinidad, (Table 1). The age range was 71 years $(21-92)$, with the age category $50-59$ years having the largest number of participants $(n=155,37.8 \%)$.

The primary result was most participants $(206,50.2 \%)$ continued to gain weight; approximately a half lost weight $(196,47.8 \%)$ and a small percentage $(8,2 \%)$ had no change in weight after the first 12 18 months of management. Twice as many women (140,68\%) gained weight compared to men $(66,32 \%)$, which may by entirely reflective of the disparity in disease occurrence among women. However only $47.1 \%(97 / 206)$ of people who gained weight had received advice on diet and exercise and 67\% (138/206) had metformin as part of their drug regimen.

\begin{tabular}{|l|c|}
\hline Sample characteristic & $\mathrm{n}(\%)$ \\
\hline Age (years) & $119(29.0)$ \\
\hline$\leq 49$ & $155(37.8)$ \\
\hline $50-59$ & $136(33.2)$ \\
\hline$\geq 60$ & $410(100)$ \\
\hline total & \\
\hline Gender & $274(66.8)$ \\
\hline female & $136(33.2)$ \\
\hline male & $410(100)$ \\
\hline total & \\
\hline Weight change & $206(50.2)$ \\
\hline Gained & $66(32)$ \\
\hline Male & $140(68)$ \\
\hline Female & $196(47.8)$ \\
\hline Loss & $67(66)$ \\
\hline Male & $129(34)$ \\
\hline Female & $8(2)$ \\
\hline No Change & \\
\hline
\end{tabular}

$\mathrm{n}=$ number of participants

Characteristics of patients in the study sample, table 1. 
All 196 (47.8\%) patients who lost weight did so by the first year of management. In other words the succeeding six months of therapy resulted in no additional weight loss. Again there were more women $(129,66 \%)$ who lost weight than men $(67,34 \%)$. It was interesting, however, that $75 \%(147 / 196)$ of participants who loss weight were in the age category $50-59$ years notwithstanding that this group represented $37.8 \%$ of the sample. The average weight loss was $3.9 \mathrm{~kg}$. The average percentage weight loss, calculated as a percentage of initial weight, was $4.5 \%$. Although more women lost weight, the average percentage weight loss was higher in males $4.9 \%$ than females $4.2 \%$ but was not statistically significant ( $\mathrm{t}$-test, $\mathrm{p}>0.05$ ) .

Further among participants who lost weight ( $\mathrm{n}=196), 69.9 \%$ were prescribed metformin. The mean weight loss of patients on metformin therapy was $4.1 \mathrm{~kg}$ and the mean percentage weight loss was $4.5 \%$. The mean weight loss for those who were not treated with metformin was $3.5 \mathrm{~kg}$ and the percentage weight loss was $4.6 \%$. In addition $45.9 \%$ also received advice on diet and exercise and this group achieved an average weight loss of $4.6 \%$ of their initial weight or an average weight reduction of $4.4 \mathrm{~kg}$.

Using the American Diabetes Association (ADA) recommended criteria of at least $5 \%$ weight loss within the first year of diagnosis and treatment $[11,20]$, only $31.6 \%(62 / 196)$ who actually loss weight attained this target or $15.1 \%(62 / 410)$ of the study population. This group consisted of $21(34 \%)$ males and $41(66 \%)$ females. Of these 62 participants, 11(17.7\%) received advice on lifestyle and exercise exclusively and achieved an average weight loss of $5.8 \mathrm{~kg}$. Of the 24 who were prescribed metformin alone achieved an average weight loss of 6.9 $\mathrm{kg}$. However those who received both advice and Metformin (19) lost an average of $11.3 \mathrm{~kg}, 8$ participants had no intervention lost an average of $7.8 \mathrm{~kg}$. While these findings may suggest that the group that had no intervention did better than the group that had advice on lifestyle only or metformin, this group was the smallest. Notwithstanding weight loss was significantly different $(\mathrm{p}=.002)$ in the group that received lifestyle, exercise and metformin $(11.3 \mathrm{Kg})$ than those who only had lifestyle and exercise intervention $(5.8 \mathrm{Kg})$.

Using multiple linear regression, the predictors of weight loss were found to be the age at which management was initiated $(\mathrm{P}=0.005$; $\beta=0.196)$ as well as the initial weight of the patient $(P=0.001 ; \beta=0.361)$. Further a significant correlation $(r=0.145, p=0.003)$ was observed between age at initial management and weight loss; that is, the older the patient the greater the percentage weight loss. The 70-79 age group had the highest average percentage weight loss of $6.8 \%$ of initial body weight.

\section{Discussion}

Among the important findings of the study was of the 647 participants with T2DM managed in a primary care setting $410(63.4 \%)$ were overweight or obese at entry into the study. Thus emphasizing that not only is overweight and obesity strongly associated with T2DM but must be prioritized as a critical component in the management of T2DM. A crucial finding was on completion of a minimum of one calendar year of therapy 196 (47\%) participants lost weight, 206 (51\%) gained weight and $8(2 \%)$ had no change in weight. The average weight loss was $0.1 \mathrm{~kg}$ or $0.1 \%$ initial body weight. Redmon et al. [22] in 2005 reported that after one year of standard therapy, which included dietary advice, an exercise regimen and other lifestyle modifications, with no drug intervention, average weight loss was roughly $0.8 \mathrm{~kg}$. [22]
Milne et al. [23] in a study of seventy diabetic volunteers who were assigned to one of three diets and measured over an 18-month period reported no weight change at all. [23] On the other hand the Diabetes Prevention Program showed that lifestyle changes and treatment with metformin both reduced the incidence of diabetes in persons at high risk. The lifestyle intervention was significantly more effective than metformin by producing 7\% weight loss. [24] Our findings therefore provide further evidence for the role of lifestyle and physical activity to be an essential component in the care of the patient with T2DM and should reinforced in the clinical setting. In addition further research is required to understand the determinants of weight loss in physician managed diabetes clinics.

Of those lost weight, 62 (31.6\%) attained the ADA recommendation of a 5-10\% loss of initial weight within the first year. [11] We were unable to find published data that examined specifically the percentage weight loss as it relates to this specific ADA recommendation. We recommend further studies on this group of patients to determine if weight loss is maintained or if patients subsequently progressed until the target is met as well as the contributing factors.

Further among those who lost weight the average weight loss was $3.9 \mathrm{~kg}$ or $4.5 \%$ of initial body weight. Gregg et al reported in 2004 in a study examining the relationship between intention to lose weight and actual weight loss in 1,401 overweight U.S. adults with diabetes reported that $45 \%$ experienced weight loss, $46 \%$ had no weight change and $9 \%$ gained weight. In addition, those with weight loss had a higher BMI, were younger, more likely to be a woman, more likely to have been hospitalized in the past year, had been diagnosed with diabetes more recently, more likely to be taking oral medications, were less likely to use insulin and more likely to report hypertension and neuropathy ( $\mathrm{p}$ $>0.05$ ). [17] We found a similar pattern in regard to gender, notably that more women lost weight compared to men but this may be explained by the larger numbers of women in the study. All participants who required drug therapy were prescribed oral hypoglycaemic agents, in other words no participants were using parenteral agents. In this regard there was a large disparity in weight gain $(51 \%)$ in our study compared to Gregg and colleagues (9\%) This large disparity in weight gain may be attributed to physician inertia in regard to weight management. It further highlights the necessity to place greater emphasis on weight loss in the management of T2DM.

The largest weight loss experienced $(11.3 \mathrm{Kg})$ was among participants who received both lifestyle interventions and metformin. Lee and Morley (1998) reported metformin to be an appetite suppressant [13] and thus a contributor to weight loss. Similarly Chaudhry et al in 2006 showed that subjects treated with metformin, or metformin and a sulfonylurea experienced a mean weight loss of 0.24 [ $\pm 0.9 \mathrm{~kg} / \mathrm{yr}]$. [25] Our findings support the combination of lifestyles interventions and metformin as an effective means of promoting weight loss in the patient with T2DM.

Weight loss in T2DM, despite its well established benefits including improved glycaemic control, has been shown to be a goal that is very difficult to achieve. [26] This is true in our setting as average weight loss of only $0.1 \%$ of initial body weight was achieved within a year of physician supervised care. Decreased energy expenditure and increased caloric retention associated with increasing glycaemic control as well as psychological factors in the patient with T2DM are some of the reasons advanced for the apparent resistance to weight loss and tendency to weight gain. [17] Weight reduction must therefore be actively 
pursued, based on sound scientific evidence as other pathological and physiological disturbances in T2DM, both by the physician and the patient.

Although lifestyle interventions should remain the cornerstone of diabetes care [22] our findings support the ADA guidelines of lifestyle interventions and metformin as the initial treatment. However, aggressive strategies such as very low-calorie diets, anti-obesity drugs and bariatric surgery have all been shown to induce significant weight loss particularly in those patients who are seemingly refractory to the more classical regimens. [27] The wider application of these interventions presents serious challenges in resource poor countries with large diabetic populations. Further in the primary care setting in the developing world, with increasing numbers of patients with T2DM it is not feasible to individualize diet and exercise regimens for each patient. We provide evidence that the existing doctor-patient consultation in its present format is ineffective in achieving evidence based guidelines for weight reduction. Gradual implementation of more aggressive strategies for those not achieving the recommended goals must be considered, such as pharmacological weight loss interventions and possibly the wider application of bariatric surgery.

The major limitations to our study included sampling from a teaching primary health care facility which may reflect a high level of care that may not be representative of all primary care facilities. We were unable to ascertain various factors that may have affected fluctuations in weight such as concurrent illness or physical conditions that may have limited the patient's ability to exercise. Gregg et al in 2004 reported that weight gain in the overweight patient with diabetes in the U.S. were more likely to be associated with worse health, and more likely to have functional limitations, although not statistically significant. [17] Other factors that may have contributed to variations in weight such as pregnancy, comorbidity or the use of medications other than metformin were similarly not taken into account. In addition, verbal communication of advice concerning dietary and lifestyle modification may not always have been documented. Similarly it was not possible to determine from patient records the extent to which the advice given was implemented. It was therefore assumed that once advice was given it was followed which may not have been the case. A final limiting factor was the unavailability of data to determine compliance with metformin.

In conclusion we have shown that the average weight loss among overweight diabetic patients in a teaching primary care facility in Trinidad is $0.1 \mathrm{~kg}$, or $0.1 \%$ of initial weight, during the first year of management. Although we found no significant association between doctor-patient counseling on lifestyle modification and weight loss or between the use of metformin only and weight loss, there was a significant association between lifestyle modifications together with metformin. Our main recommendations are the recognition and prioritization of weight loss as an equal component among other targets in T2DM, as well as the development of structured programs dedicated to weight reduction.

\section{References}

1. American Diabetes Association (2011) Diagnosis and classification of diabetes mellitus. Diabetes Care 34: S62-S69.

2. World Health Organization Fact Sheet $N^{\circ} 312$ [Online]. 2006 Sep: [cited 2008 Mar 5].

3. Haslam DW, James WP (2005) Obesity. Lancet 366: 1197-1209.

4. Miller GJ, Maude GH \& Beckles GL (1996) Incidence of hypertension and noninsulin dependent diabetes mellitus and associated risk factors in a rapidly developing Caribbean community: the St James survey, Trinidad. J Epidemio Community Health 50: 497-504.

5. Pan American Health Organization/World Health Organization (PAHO/WHO) Leading Causes of Death And Mortality Rates (Counts and Rates) in Caribbean Epidemiology Centre Member Countries $1^{\text {st }}$ Ed. Port of Spain: Publications of the Caribbean Epidemiology Centre (CAREC) 2005: 24.

6. Barceló Alberto, Aedo Cristian, Rajpathak Swapnil, Robles Sylvia (2003) The cost of diabetes in Latin America and the Caribbean. Bull World Health Organ [serial on the Internet]. 81: 19-27.

7. Wagner E, Sandhu N, Newton K, Mc Culloch D, Ramsey S, Grotheus L (2001) Effect of Impaired Glycemic control on Health Care costs and Utilisation. JAMA 285:182-189.

8. Gulliford MC, Mahabir D (2002) Diabetic foot disease and foot care in a Caribbean community. Diabetes Research Clinical Practice 56: 35-40.

9. The Diabetes Control and Complications Trial Research Group (1993) The effect of intensive treatment of diabetes on the development and progression of long-term complications in insulin-dependent diabetes mellitus. N Engl J Med 329: 977-986.

10. The UK Prospective Diabetes Study (UKPDS) Group (1998) Effect of intensive blood-glucose control with metformin on complications in overweight patients with type 2 diabetes (UKPDS 34). Lancet 352: 854-865.

11. Standards of Medical Care-2011.American Diabetes Association. Diabetes Care 2010: 34 S11-S61.

12. Boyne M, Carter A, Doobay R, Forde L, Fraser H, Goddard C, et al. (2006) Managing Diabetes In Primary Care In The Caribbean. Caribbean Health Research Council and PAHO.

13. Lee A, Morley JE (1998) Metformin Decreases Food Consumption and Induces Weight Loss in Subjects with Obesity with Type 2 Non-Insulin Dependant Diabetes. Obes Res 6: 47-53.

14. Avenell A, Broom J, Brown TJ, Poobalan A, Aucott L, et al. (2004) Systematic review of the long-term effects and economic consequences of treatments for obesity and implications for health improvement. Health Technol Assess 8: $1-182$.

15. Hussain P, Kawar B, El Nahas M (2007) Obesity and diabetes in the developing world - a growing challenge. N Engl J Med 356: 213-215.

16. World Health Organization (2000) Obesity: preventing and managing the global epidemic. Geneva: WHO

17. Gregge E, Gerzoff R, Thompson T, Williamson D (2004) Trying to Lose Weight Losing Weight and 9 Year Mortality in Overweight U.S. Adults With Diabetes Mellitus. Diabetes Care 27: 1499-1504.

18. American Diabetes Association, the North American Association for the Study of Obesity (2005). Weight Management Using Lifestyle Modification in the Prevention and Management of Type 2 Diabetes: Rationale and Strategies. Clinical Diabetes 23: 130-133.

19. Aucott L, Poobalan A, Smith WCS, Avenelle A, Jung R, et al. (2004) Weight Loss in Obese Diabetic and Non-Diabetic Individuals ad Long Term Diabetes Outcomes-a Systematic Review. Diabetes, Obesity and Metabolism 6: 85-94.

20. Noel PH, Pugh JA (2002) Management of Overweight and Obese Adults. BM 325: 757-761.

21. Trinidad and Tobago Diabetes Guide (2011).

22. Redmon JB, Raatz SK, Reck KP, Swanson JE, Kwong CA, et al. (2003) One Year Outcome of a Combination of Weight Loss Therapies for Subjects with Type 2 Diabetes. Diabetes Care 26: 2505-2511.

23. Milne RM, Mann JI, Chrisholm AW, Willams SM (1994) Long-term Comparison of Three Dietary Prescriptions in the Treatment of NIDDM. Diabetes Care 17 74-80.

24. Knowler WC, Barrett-Connor E, Fowler SE, Hamman RF, Lachin JM et al. (2002) Reduction in the Incidence of Type 2 Diabetes with Lifestyle Intervention or Metformin. N Engl J Med 346393-403.

25. Chaudry ZW, Gannon MC, Nuttall FQ (2006) Stability of Body Weight in Type 2 Diabetics. Diabetes care 29: 493-497. 
Citation: Mungrue K, Roper LA, Chung T (2011) Assessment of Weight Loss in the Management of Patients with Type 2 Diabetes Mellitus in Primary Care in Trinidad. J Diabetes Metab 2:120. doi:10.4172/2155-6156.1000120

Page 5 of 5

26. Hensrud DD (2001) Dietary Treatment and Long-Term Weight Loss and Maintenance in Type 2 Diabetes. Obes Res 9: 348S-353S
27. Scheen AJ (1998) Aggressive Weight Reduction Treatment in the Management of Type 2 Diabetes. Diabetes Metab 24: 116-123. 